\title{
Evaluation of in vivo Antiplasmodial Activities of extracts of Morinda morindiodes (Bak.) in the treatment of malaria in Ogun State
}

\author{
Soniran O Temidayo*, Idowu Olufunmilayo, Idowu A Babatunde, Ajana Olusegun \\ From Parasite to Prevention: Advances in the understanding of malaria \\ Edinburgh, UK. 20-22 October 2010
}

In vivo study of various plant parts extracts of Morinda morindiodes (Bak.) was conducted to evaluate their antiplasmodial properties and effects on the liver using chloroquine sensitive Plasmodium berghei in mice. Water extract of the root was observed to significantly reduce parasitaemia $(70 \%, \mathrm{P}<0.05)$ compared to the activities of other plant parts and the untreated control. A mean survival time of 19 days observed in the root extract supported its antiplasmodial activities compared with other plant parts. The antiplasmodial activities of the plant extracts when administered twice daily were not significantly different
$(\mathrm{P}>0.05)$ compared with those treated once daily. The chemosuppression produced by the extracts were significantly different compared to untreated control. Liver function tests (LFT) of uninfected mice administered with the plant extracts showed that extracts of the leaf and stem in 'fermented maize starch extract' altered the function of the liver significantly compared to normal mice. This study shows that Morinda morindiodes possess antimalarial properties and the root may be used as a prophylaxis where western medicine is not easily accessible and affordable. Tables 1, 2, 3, 4

Table 1 Chemosuppression and survival time of $P$. berghei infected mice treated orally with Morinda morindiodes extracts at a dose of $100 \mathrm{mg} / \mathrm{kg}$ body weight once a day for 5 days

\begin{tabular}{|c|c|c|c|c|}
\hline Plant part & Extract & $\begin{array}{l}\text { \%chemosuppression of parasitaemia at day } 5 \\
\text { (C.P. } \pm \text { S.D) }\end{array}$ & $\begin{array}{l}\text { \%chemosuppression of parasitaemia at day } \\
\qquad 11 \text { (C.P. } \pm \text { S.D) }\end{array}$ & $\begin{array}{l}\text { Mean Survival time } \\
\text { (days) }\end{array}$ \\
\hline \multirow[t]{3}{*}{ Leaf } & $\mathrm{MeOH}$ & $7.5 \pm 2.8^{\mathrm{b}}$ & $82.4 \pm 5.6^{\mathrm{e}}$ & 19.5 \\
\hline & Water & $10 \pm 2.8^{b c}$ & $72.6 \pm 8.4^{\mathrm{bc}}$ & 16.5 \\
\hline & $\begin{array}{l}\text { F.M. } \\
\text { starch }\end{array}$ & $7.5 \pm 2.8^{b}$ & $78.8 \pm 9.9^{d}$ & 16.5 \\
\hline \multirow[t]{3}{*}{ Stem } & $\mathrm{MeOH}$ & $0 \pm 2.8^{\mathrm{a}}$ & $60.8 \pm 5.6^{a}$ & 16.5 \\
\hline & Water & $1.3 \pm 1.41^{\mathrm{ab}}$ & $72.2 \pm 14.8^{\mathrm{bc}}$ & 15.5 \\
\hline & $\begin{array}{l}\text { F.M. } \\
\text { starch }\end{array}$ & $5.0 \pm 2.8^{b}$ & $71.6 \pm 0.0^{\mathrm{bc}}$ & 16.5 \\
\hline \multirow[t]{3}{*}{ Root } & $\mathrm{MeOH}$ & $20.5 \pm 22.6^{\mathrm{bc}}$ & $67.3 \pm 7.07^{\mathrm{b}}$ & 18.5 \\
\hline & Water & $52.5 \pm 2.8^{\mathrm{d}}$ & $76.5 \pm 0.0^{\mathrm{cd}}$ & 19.5 \\
\hline & $\begin{array}{l}\text { F.M. } \\
\text { starch }\end{array}$ & $30 \pm 8.5^{c}$ & $79.1 \pm 0.0^{d}$ & 19.5 \\
\hline Chloroquine & & & $100 \pm 0.0^{e}$ & 28.5 \\
\hline Artesunate & & & $100 \pm 0.0^{e}$ & 28.5 \\
\hline Control & & & 0.0 & 14.5 \\
\hline
\end{tabular}

MeOH, methanol extract; F.M. starch, "aqueous fermented maize starch ('omidun') extract".

Department of Biological Sciences, University of Agriculture, Abeokuta 
Table 2 Chemosuppression and survival time of $\boldsymbol{P}$. berghei infected mice treated orally with Morinda morindiodes extracts at a dose of $100 \mathrm{mg} / \mathrm{kg}$ body weight twice a day for 5 days

\begin{tabular}{|c|c|c|c|c|}
\hline Plant part & Extract & $\begin{array}{l}\text { \%chemosuppression of parasitaemia at day } 5 \\
\text { (C.P. } \pm \text { S.D) }\end{array}$ & $\begin{array}{l}\text { \%chemosuppression of parasitaemia at day } \\
11 \text { (C.P. } \pm \text { S.D) }\end{array}$ & $\begin{array}{l}\text { Mean Survival time } \\
\text { (days) }\end{array}$ \\
\hline \multirow[t]{3}{*}{ Leaf } & $\mathrm{MeOH}$ & $20 \pm 16.9 a$ & $83.7 \pm 8.5^{c}$ & 21.5 \\
\hline & Water & $5.0 \pm 5.6^{\mathrm{ab}}$ & $76.1 \pm 1.4^{\mathrm{bc}}$ & 17.5 \\
\hline & $\begin{array}{l}\text { F.M. } \\
\text { starch }\end{array}$ & $27.5 \pm 28^{\mathrm{abc}}$ & $79.4 \pm 24.1^{b c}$ & 17.5 \\
\hline \multirow[t]{3}{*}{ Stem } & $\mathrm{MeOH}$ & $1.3 \pm 11.3^{\mathrm{a}}$ & $73.2 \pm 25.4^{b}$ & 16.5 \\
\hline & Water & $2.5 \pm 28.2^{\mathrm{a}}$ & $71.2 \pm 2.8^{\mathrm{b}}$ & 16.5 \\
\hline & $\begin{array}{l}\text { F.M. } \\
\text { starch }\end{array}$ & $10.0 \pm 5.6^{\mathrm{ab}}$ & $76.5 \pm 9.9^{b c}$ & 17.5 \\
\hline \multirow[t]{3}{*}{ Root } & $\mathrm{MeOH}$ & $35 \pm 5.6^{b c}$ & $80.4 \pm 28.2^{b c}$ & 19.5 \\
\hline & Water & $70.0 \pm 2.8^{\mathrm{de}}$ & $85.9 \pm 8.4^{\mathrm{bc}}$ & 21.5 \\
\hline & $\begin{array}{l}\text { F.M. } \\
\text { starch }\end{array}$ & $56.2 \pm 7.1^{\mathrm{cd}}$ & $85.6 \pm 8.4^{c}$ & 17.5 \\
\hline Chloroquine & & & $100 \pm 0.0^{e}$ & 28.5 \\
\hline Artesunate & & & $100 \pm 0.0^{\mathrm{e}}$ & 28.5 \\
\hline Control & & & 0.0 & 14.5 \\
\hline
\end{tabular}

$\mathrm{MeOH}$, methanol extract; F.M. starch, "aqueous fermented maize starch ('omidun') extract".

Table 3 Comparison between the liver function tests in mice treated with extracts and control group (untreated)

\begin{tabular}{|c|c|c|c|c|c|c|c|}
\hline \multicolumn{2}{|l|}{ Leaf extracts } & \multicolumn{2}{|c|}{$\begin{array}{c}\text { Stem } \\
\text { extracts }\end{array}$} & \multicolumn{2}{|c|}{$\begin{array}{c}\text { Root } \\
\text { extracts }\end{array}$} & \multicolumn{2}{|c|}{ Contrl } \\
\hline Test & WL & F.M.L & WS & F.M.S & WR & F.M.R & INT. \\
\hline \multicolumn{8}{|l|}{$\begin{array}{l}\text { Treated Once } \\
\text { Daily }\end{array}$} \\
\hline Total protein(g/l) & $53^{\mathrm{a}}$ & $58.8^{b}$ & $61.7^{c}$ & $62.4^{\mathrm{cd}}$ & $63.5^{\mathrm{cd}}$ & $52.8^{\mathrm{a}}$ & $65.1 \mathrm{~d}$ \\
\hline $\begin{array}{l}\text { Cholesterol (mg/ } \\
\text { dl) }\end{array}$ & $88.7^{b}$ & $102.3^{d}$ & $106.9^{e}$ & $95.2^{c}$ & $87.2^{\mathrm{b}}$ & $63.6^{\mathrm{a}}$ & $106.9 \mathrm{e}$ \\
\hline SGOT (iu/l) & $66^{c}$ & $67^{c}$ & $85^{d}$ & $91^{\mathrm{e}}$ & $67^{c}$ & $30^{\mathrm{a}}$ & $44 b$ \\
\hline SGPT (iu/l) & $17^{\mathrm{ab}}$ & $20^{\mathrm{b}}$ & $18^{\mathrm{b}}$ & $13^{\mathrm{a}}$ & $28^{c}$ & $25^{c}$ & $27 c$ \\
\hline Urea (mg/dl) & $24.5^{\mathrm{a}}$ & $28.35^{c}$ & $25.7^{\mathrm{ab}}$ & $26.5^{\mathrm{bc}}$ & $26.5^{\mathrm{bc}}$ & $28.35^{c}$ & $28 \mathrm{c}$ \\
\hline $\begin{array}{l}\text { Alkaline } \\
\text { phosphatase (iu/l) }\end{array}$ & $95^{e}$ & $80^{d}$ & $78^{c}$ & $47^{\mathrm{ab}}$ & $40^{a}$ & $62 b$ & \\
\hline \multicolumn{8}{|l|}{$\begin{array}{l}\text { Treated twice } \\
\text { Daily }\end{array}$} \\
\hline Total protein(g/l) & $60.8^{c}$ & $50.4^{b}$ & $76.7^{e}$ & $46^{\mathrm{a}}$ & $75.5^{\mathrm{e}}$ & $65.9^{d}$ & $65.1 d$ \\
\hline $\begin{array}{l}\text { Cholesterol (mg/ } \\
\text { dl) }\end{array}$ & $98.2^{f}$ & $75.4^{b}$ & $83.6^{c}$ & $92.7^{e}$ & $60.9^{\mathrm{a}}$ & $87.1^{d}$ & $106.9 \mathrm{~g}$ \\
\hline SGOT (iu/l) & $43^{c}$ & $92^{d}$ & $36^{\mathrm{b}}$ & $93^{d}$ & $35^{\mathrm{b}}$ & $19^{\mathrm{a}}$ & $44 c$ \\
\hline SGPT (iu/l) & $23^{c}$ & $22.5^{c}$ & $15^{\mathrm{ab}}$ & $23^{c}$ & $12^{\mathrm{a}}$ & $17^{\mathrm{b}}$ & $27 d$ \\
\hline Urea (mg/dl) & $22.5^{\mathrm{a}}$ & $25.05^{\mathrm{b}}$ & $27.3^{c}$ & $25.1^{b}$ & $24.2^{\mathrm{ab}}$ & $24.2^{\mathrm{ab}}$ & $28 C$ \\
\hline $\begin{array}{l}\text { Alkaline } \\
\text { phospatase (iu/l) }\end{array}$ & $29^{a}$ & $58^{d}$ & $63^{d}$ & $37^{\mathrm{b}}$ & $34^{\mathrm{ab}}$ & $60^{c d}$ & $62 d$ \\
\hline
\end{tabular}

WL, water extract of leaf; F.M. L, aqueous fermented maize starch extract of leaf.
Table 4 Phytochemical analysis of the various plant parts of Morinda morindiodes

\begin{tabular}{lcccc}
\cline { 1 - 2 } \multicolumn{3}{c}{ Plant Parts / Quantity of Compound } & \\
\cline { 1 - 3 } Investigated Compounds & Leaf & Stem & Root \\
\hline Alkaloid (g/100g) & 1.42 & 1.96 & 1.62 \\
Saponin (g/100g) & 25.3 & 26.1 & 22.5 \\
Tannin (mg/100g) & 46.2 & 49.2 & 38.55 \\
Flavonoid (mg/100g) & 14.2 & 10.4 & 12.1 \\
Glycocyanides (mg/100g) & $\mathbf{0 . 9 8}$ & 1.06 & 1.12 \\
\hline
\end{tabular}

Published: 20 October 2010

\section{References}

1. Abosi AO, Raseroka BH: In vivo antimalarial activity of Vernonia amygdalina. British Journal of Biomedical Science 2003, 60(22):89-91.

2. Ajaiyeoba EO, Oladepo O, Fawole OI, Bolaji OM, Akinboye DO, Ogundahunsi OAT, Falade CO, Gbotosho GO, Itiola OA, Happi TC, Ebong OO, Ononimu IM, Osowole OS, Oduola OO, Ashidi JS, Oduola AMJ: Cultural categorization of febril illnesses in correlation with herbal remedies used for treatment in Southwestern Nigeria. Journal of Ethnopharmacology 2003, 85:179-185.

3. Andrade-Neto VF, Brandao MG, Stehman JR, Oliveira LA, Krettli AU: Antimalarial activity of Cinchona-like plants used to treat fever and malaria in Brazil. Journal of Ethnopharmacology 2003, 87:253-256.

4. Harborne JB: Phytochemical Methods. A Guide to Modern Techniques to Plant Analysis London, Chapman and Hall, 3rd edn 1987, 56-79.

5. Idowu OA, Soniran OT, Ajana O, Aworinde DO: Ethnobotanical Survey of antimalarial plants used in Ogun State, Southwest Nigeria. African Journal of Pharmacy and Pharmacology 2010, 4:55-60.

doi:10.1186/1475-2875-9-S2-P51

Cite this article as: Temidayo et al:: Evaluation of in vivo Antiplasmodial Activities of extracts of Morinda morindiodes (Bak.) in the treatment of malaria in Ogun State. Malaria Journal 2010 9(Suppl 2):P51. 